\title{
Robust Trajectory Optimization Under Frictional Contact with Iterative Learning
}

\author{
Jingru Luo \\ School of Informatics and Computing \\ Indiana University Bloomington \\ Bloomington, IN, USA \\ Email: luojing@indiana.edu
}

\author{
Kris Hauser \\ Department of Electrical and Computer Engineering \\ Department of Mechanical Engineering and Materials Science \\ Duke University \\ Durham, NC, USA \\ Email: kris.hauser@duke.edu
}

\begin{abstract}
Optimization is often difficult to apply to robots due to the presence of modeling errors, which may cause constraints to be violated during execution on a real robot. This work presents a method to optimize trajectories with large modeling errors using a combination of robust optimization and parameter learning. In particular it considers the problem of computing a dynamically-feasible trajectory along a fixed path under frictional contact, where friction is uncertain and actuator effort is noisy. It introduces a robust time-scaling method that is able to accept confidence intervals on uncertain parameters, and uses a convex parameterization that allows dynamically-feasible motions under contact to be computed in seconds. This is combined with an iterative learning method that uses feedback from execution to learn confidence bounds on modeling parameters. Experiments on a manipulator performing a "waiter" task, on which an object is moved on a carried tray as quickly as possible, demonstrate this method can compensate for modeling uncertainties within a handful of iterations.
\end{abstract}

\section{INTRODUCTION}

Optimal motions are hard to realize on robots because there is a fundamental tradeoff between optimality and robustness. Optimal trajectories pass precisely at the boundary of feasibility, so errors in the system model or disturbances in execution will usually cause feasibility violations. For example, optimal motions in the presence of obstacles will cause the robot to graze an object's surface. It is usually impossible or impractical to obtain extremely precise models, particularly of dynamic effects, such as inertial parameters and friction coefficients. In legged locomotion, such errors may cause a catastrophic fall, and in nonprehensile manipulation, such errors may cause the object to slip, tip, or fall. Moreover, accurate execution of planned trajectories is difficult on hardware, particularly as the field of robotics progressively adopts compliant, human-safe actuators that are less precise than the highly-geared electric motors of traditional industrial robots. A final source of error is numerical error in optimization algorithms, such as the resolution of constraint checks using pointwise collocation. One way to increase robustness is to add a margin of error to optimization constraints, e.g., by assuming very small frictions, conservative velocity bounds, or collision avoidance margins. However, this leads to unnecessarily slow executions and difficulties in tuning appropriate margins.

This paper presents an iterative learning approach in which a robot 1) learns the errors in its models given execution
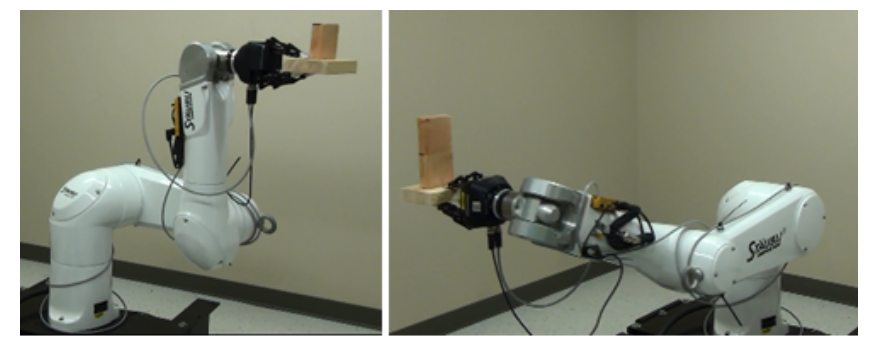

Fig. 1: Left: The waiter task for a single block. Right: the task for a stack of two blocks.

feedback, 2) incorporates estimated errors into optimization, and 3) repeats the process until it converges to successful and/or near-optimal executions. Specifically, it addresses tasks under frictional contact with significant friction uncertainty and execution errors, such as legged locomotion and object manipulation tasks, and its main contributions lie in a robust trajectory optimization module and an execution feedback module. The robust optimization accepts confidence intervals on the uncertain parameters and computes a dynamicallyfeasible time-parameterized trajectory along a fixed geometric path with contact constraints. Observations from execution are used to adjust the intervals in the optimization iteratively to achieve robustness.

Our contributions help the learning process proceed at interactive rates. Although trajectory optimization is widely used in robotic motion planning and optimal control, optimization under frictional contact remains challenging. For tractability of computation we consider optimizing only velocity along a fixed path given dynamic constraints (the time-scaling problem). This paper formulates a new fast time-scaling method for generating an optimal, robust, dynamically feasible timeparameterized trajectory along a fixed geometric path for a robot in frictional contact. We formulate the contact constraints on the system as a linear program that is solved at each constraint evaluation point of the time-scaling optimization. This transforms the optimization problem into a two level hierarchical optimization with the confidence intervals on uncertain parameters working as margins in both levels. The decomposed optimization runs much faster (in seconds) than the monolithic large optimization (in minutes). 
We test our methods on a manipulator performing a "waiter" task (Fig. 1). Imagining a robotic waiter serving customers, objects are placed on a tray carried by the robot and the goal is to move the objects as quickly as possible without causing them tipping over. Modeling and execution uncertainties are inevitable. For irregular-shaped objects and objects made from unknown materials, it is difficult to have the precise values on the contact friction and COM of the objects. Moreover, there could be large disturbances acting on the tray in a dynamic environment similar to a busy restaurant. Using the proposed framework, the robot would "practice" a handful of times to learn estimates of parameter and execution uncertainty, and then maintain those estimates to optimize trajectories robustly in the future.

We handle two types of uncertainties: (i) parameter estimation errors for parameters of constant value over time, such as coefficient of friction (COF), hardware calibration, contact points position, center of mass (COM) of a manipulated object, and (ii) execution errors from control and disturbances. These are handled via two types of execution feedback:

1) Binary execution feedback (success or failure) is used to estimate unobservable parameters of the dynamic model. Our method accepts estimated ranges of the chosen parameter, and then uses a bisection search method to determine whether we have over- or under-estimated its value, converging to a value that leads to a feasible execution within $\epsilon$ of optimal.

2) Joint sensor feedback on the executed trajectory lets us estimate execution errors (e.g., random disturbances). We construct confidence intervals on the deviation between executed and planned trajectory as margins into optimization.

We can consider both error types either individually or simultaneously. In the latter case, we learn both modeling and execution errors in an iterative fashion.

Experiments evaluate our method in simulation and on hardware with an industrial manipulator, Stäubli TX90L manipulating blocks (Fig 1). The results demonstrate our robust trajectory optimization can generate dynamically-feasible trajectories within 10 seconds on a standard PC, and the iterative learning approach is able to produce robust task executions within a handful of iterations.

\section{RELATED WORK}

The problem of generating time-optimal, dynamicallyfeasible trajectory of a given path without considering contact was initially solved by Bobrow et al [4] and Shin and McKay [26] and later enhanced by [7, 8, 18, 27]. Recent work has extended the time-scaling problem to handle frictional contact [15]. We further extend the time-scaling concept to incorporate robustness margins in friction parameters and execution disturbances.

Although the problem of time-optimal path parameterization is well-studied for robots operating in free-space [3, 29], this problem has not been widely considered for robots that make or break contact. Several authors have considered optimizing both joint trajectories and timing for legged robots [9, 12, 23, [24]. A trajectory optimization in Cartesian space is presented in [12] for solving humanoid motions and is suitable for generating motion primitives. In [9], a contact-before-motion planner which grows a search tree using a rough trajectory is proposed. [23, 24] present a direct trajectory optimization method which formulates the reaction forces as optimization parameters. But due to the size of the optimization problem, these techniques are often extremely slow, taking minutes or hours to complete. Some numerical approaches have been presented for dexterous manipulation [17, 20] as well as nonprehensile grasp [16, 19, 22], and they are computationally challenging to be extended to high dimensional problems.

Recent work [15] considered contact forces and presented a fast convex time-scaling method with polytope projection techniques to precompute dynamic feasible sets. Similar to [15], our work avoids the computation of exact contact forces to speed up the optimization. But we use a different formulation on the contact constraints through a transformation into smooth linear programs at constraint evaluation points, which allows us to consider robustness to execution errors without severely increasing running time. We also illustrate how to incorporate the coupled dynamic constraints between manipulated objects as well as the robot.

Robotic applications cannot escape uncertainties in terms of imperfect system modeling and robot control as well as unknown execution disturbances. Many learning methods have been proposed to handle uncertainties in different levels: learning from demonstration [1] for computing a task-specific policy, model learning with machine learning techniques [21] and control learning [5, 25]. In this work, we utilize ideas from previous work and use an iterative learning approach based on a binary search method and a robust trajectory optimization to learn the uncertainties from modeling and execution. The robust optimization models acceleration errors as range estimation (similar to [2]) and optimizes a nonlinear problem to generate a trajectory that is robust up to a given confidence value.

\section{PRoblem Formulation}

Our goal is to generate a robust, dynamically-feasible, and near-optimal trajectory for a robot manipulating an object under frictional contact and imperfect knowledge about the system model and random execution errors. The robot is allowed to execute trajectories repeatedly to improve its estimates. However, its feedback will only consist of the executed joint trajectories and success/failure of the manipulation; no external observations like object motion capture or pressure sensors are available.

For simplicity we assume that a geometric path (without timing information) is given. In future extensions of this work, the shape of the path may be optimized as well. We also restrict ourselves to two types of errors: modeling errors in the coefficient of friction, and random disturbances in the motion execution, although our work may be applicable to other errors as well. 


\section{A. Summary of method}

The method operates as follows:

1) The robot is given a geometric path, robot-object contact points, and initial confidence intervals of the uncertainty in modeling parameters and execution errors. (These may be pessimistic or optimistic; they will be adapted later via execution feedback.)

2) The robust time-scaling optimization is applied to generate a trajectory that is feasible under the given confidence intervals.

3) The optimized path is executed. Success / failure and sensing feedback are used to improve the confidence intervals.

4) Steps 2 and 3 are iterated until success and/or a desired level of convergence is achieved.

\section{B. Robot Motion Constraints}

Given a twice-differentiable geometric path $q(s): s \in$ $[0,1] \mapsto R^{n}$ for a robot of $n$ degrees of freedom (DOFs), we wish to find a time parameterization $s(t): t \in\left[0, t_{f}\right] \mapsto[0,1]$ and a final time $t_{f}$ such that an objective function $f_{o b j}$ is minimized and a set of constraints is satisfied.

The objective function $f_{o b j}$ considered here is the minimum execution time but can also include other terms such as minimum jerk or energy. The set of constraints includes velocity, acceleration and torque limits given by:

$$
\begin{aligned}
& \dot{q}_{\text {min }} \leq \dot{q} \leq \dot{q}_{\max } \\
& \ddot{q}_{\min } \leq \ddot{q} \leq \ddot{q}_{\max } \\
& \tau_{\min } \leq \tau \leq \tau_{\max }
\end{aligned}
$$

Also, for safety reasons, we usually require the robot starts and ends at a stop:

$$
\begin{aligned}
& \dot{q}(0)=0 \\
& \dot{q}(1)=0
\end{aligned}
$$

We are interested in obtaining dynamically-feasible motion with robot in contact. In addition to the geometric path $q(s)$, we assume the input also contains the following information:

1) If there exist $N_{p}>1$ contact phases, the domain of $q$ is divided into sections $s p_{0}=0, s p_{1}, \ldots, s p_{N_{p}}=$ $1\}$ in which contacts are constant over the range $\left\{q(u) \quad \mid s p_{i}<u<s p_{i+1}\right\}$.

2) The set $F_{i}$ of contact points and normals in each phase $i=1, \ldots, N_{p}$.

For simplicity, we describe our formulation and methods in the simplest case which only has one contact phase and the handling of multiple contact phases is presented in section IV-E

The contact enforces extra constraints: contact forces are limited within friction cones and maintaining the contact states indicates zero velocity of contact points in world space. Let $f_{1}, \ldots, f_{m}$ be the contact forces at points $p_{1}, \ldots, p_{m}$ respectively. The system dynamics is given by:

$$
M(q) \ddot{q}+C(q, \dot{q})+G(q)=\tau+\sum_{i} J_{i}(q)^{T} f_{i}
$$

where $M$ is the mass matrix, $C$ is the centrifugal and Coriolis forces which are quadratic in the joint velocities, and $G$ is the generalized gravity vector, $J_{i}(q)$ is the Jacobian of point $p_{i}$ with respect to robot's configuration $q$. We assume that the initial path is chosen to respect the condition that fixed contact positions have zero velocity in world space: $J_{i}(q) \frac{d q}{d s}=$ 0 . Also, we consider the friction constraints $f_{i} \in F C_{i}$ for $i=1, \ldots, m$, where $F C_{i}$ is the friction cone at contact position $p_{i}$. For the purposes of this paper, we assume all friction cones are convex and we approximate the nonlinear cones by a set of linear constraints expressed as:

$$
A_{i} f_{i} \leq 0 \text { for } i=1, \ldots, m
$$

Since each friction cone is dependent on a coefficient of friction estimate $\mu_{i}$ we may write the dependence explicitly as $F C_{i} \equiv F C_{i}\left(\mu_{i}\right)$ and $A_{i} \equiv A_{i}\left(\mu_{i}\right)$.

\section{Extra Constraints for the Object Manipulation Task}

For the "waiter" task, to move the objects together with the robot, we must ensure the linear acceleration $\alpha_{L}$ and angular acceleration $\alpha_{A}$ of the object can be supported by the contact forces and gravity, therefore the Newton-Euler equations impose two dynamic constraints on the system:

$$
\begin{aligned}
m_{o b j} \alpha_{L} & =\sum_{i} f_{i}+m_{o b j} g \\
I_{o b j} \alpha_{A} & =\sum_{i}\left(p_{i}-c\right) \times f_{i}
\end{aligned}
$$

where $m_{o b j}$ and $I_{o b j}$ are the mass and inertia matrix of the block respectively, $p_{i}$ is the contact point, and $c$ is the center of mass (all quantities given in world coordinates). Given the configuration, velocity and acceleration of the robot, the accelerations of the object can be computed through the Jacobian and Hessian of the object's pose in the robot frame:

$$
\left(\begin{array}{c}
\alpha_{L} \\
\alpha_{A}
\end{array}\right)=J(q) \ddot{q}+H(q) \dot{q}^{2}
$$

\section{Robust TIME-SCALING WITH FRICTION}

This section presents a robust time-scaling optimization method. Computation speed is improved by transforming the constraints on the system dynamics and contact forces into a linear program so that the problem size is reduced to be independent of the number of contact points. Robustness against execution errors is achieved by optimizing over a confidence interval which is an estimation of the noise on the execution acceleration.

\section{A. Robust Motion Constraints with Confidence Intervals}

Errors introduce nontrivial changes in the way dynamic constraints are evaluated; for example in the "waiter" task, the executed acceleration of the object, rather than the planned acceleration, decides if the contact forces are sufficient to move the blocks without tipping over. Moreover, the object's acceleration is determined in a nontrivial manner by the robot's velocity and acceleration. 


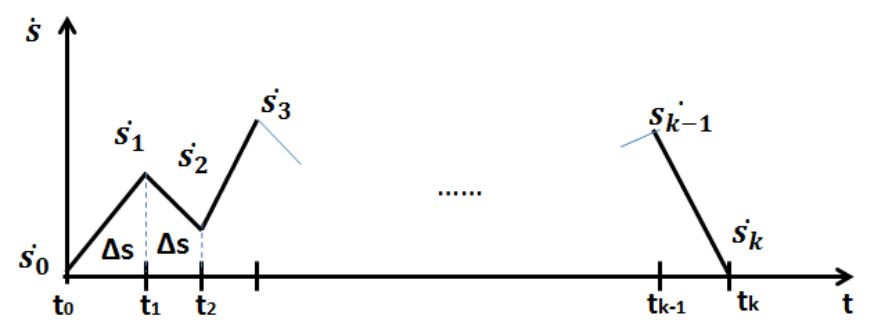

Fig. 2: Velocity $\dot{s}$ of the time scaling.

Errors on robot's execution velocity and acceleration can be modeled as joint-wise confidence intervals $\dot{q}-\dot{q}_{\text {plan }} \in$ $\left[\dot{q}_{l o w}, \dot{q}_{u p p}\right]$ and $\ddot{q}-\ddot{q}_{\text {plan }} \in\left[\ddot{q}_{\text {low }}, \ddot{q}_{\text {upp }}\right]$ where $\dot{q}_{\text {plan }}$ is the planned velocity and $\ddot{q}_{\text {plan }}$ is the planned acceleration. For simplicity, our method ignores the errors in $\dot{q}$ because the errors are usually two orders of magnitude smaller than acceleration errors.

As described in later sections, we will estimate joint-wise execution errors as $\ddot{q}_{e x c}=\ddot{q}_{\text {plan }}+e$ where $e$ is subject to Gaussian distribution $N\left(U, \Delta^{2}\right)$ to account for the uncertainties from execution and unknown disturbances. We incorporate them into the optimization as follows:

$$
\begin{aligned}
& \ddot{q}_{\text {upp }}=U+K \cdot \Delta \\
& \ddot{q}_{\text {low }}=U-K \cdot \Delta
\end{aligned}
$$

where $K$ controls the confidence ratio on uncertainties.

When we apply the confidence interval to the dynamic constraints, we wish to ensure that all constraints related to $\ddot{q}$ in (2), (5) and (8) are satisfied with respect to the execution accelerations. As a result, this imposes additional upper and lower constraints on the planned accelerations.

For friction coefficient confidence intervals $\left[\mu_{l o w}, \mu_{u p p}\right]$, it is clear that we only need to formulate the lower end of the confidence interval $\mu_{\text {low }}$ because any solution for the lower friction case is also a solution for the higher friction case.

\section{B. Time-Scaling Function}

The time scaling method extends the method of [15] to handle execution errors. The constraints on the velocity and acceleration require the time-scaling function $s(t)$ to be at least twice differentiable. So we define the velocity of the path $s(t)$ as a piecewise linear interpolation between $\dot{S}=\left\{\dot{s_{0}}, \ldots, \dot{s_{N}}\right\}$ (see Fig. 2) and then $s(t)$ becomes a piecewise quadratic curve consisting of $N$ segments $s_{i}(t), i=1, \ldots, N$ with constant acceleration in each segment. We also assume each segment as a uniform interval $\Delta s$ to simplify the computation. Therefore, the objective function of minimizing execution time is:

$$
f_{o b j}(\dot{S})=\sum_{i=0}^{N} \frac{\Delta s}{\dot{s}_{i}+s_{i+1}}
$$

Using interval arithmetic notation, we can express the executed derivatives as follows:

$$
\dot{q}(s, \dot{s}) \in \frac{d q}{d s} \dot{s}+\left[\dot{q}_{l o w}, \dot{q}_{u p p}\right]
$$

$$
\ddot{q}(\dot{s}, \ddot{s})=\frac{d^{2} q}{d s^{2}} \dot{s}^{2}+\frac{d q}{d s} \ddot{s}+\left[\ddot{q}_{l o w}, \ddot{q}_{u p p}\right] .
$$

Since we assume negligible velocity error, the system dynamics $\sqrt{5}$ can be represented in terms of $\dot{s}, \ddot{s}$ as follows:

$a(s) \ddot{s}+b(s) \dot{s}^{2}+c(s)=\tau+\sum_{i} J_{i}(q)^{T} f_{i}-M(q)\left[\ddot{q}_{l o w}, \ddot{q}_{u p p}\right]$

where $a(s)=M(q) \frac{d q}{d s}, b(s)=M(q) \frac{d^{2} q}{d s^{2}}+C\left(q, \frac{d q}{d s}\right)$ and $c(s)=G(q)$. The last term on the right hand size involves a product of a matrix and a vector interval, and is computed using interval arithmetic.

The optimization can now be performed over a time parameterization $s(t)$, with constraints (1- 3) and (7) enforced at discretized values $s_{1}, \ldots, s_{N}$ along the $s$ coordinate. Note that significant speed gains can be achieved by computing the coefficients $\frac{d q}{d s}(s), \frac{d^{2} q}{d s^{2}}(s), a(s), b(s), c(s)$ only once for all discretized values of $s$.

\section{Hierarchical Optimization}

We discretize the constraints along the optimized trajectory by enforcing them at $N$ collocation points $([13])$. To solve this problem, a naïve method is to parameterize both the robot trajectory expressed in terms of $\dot{s}, \ddot{s}$ as in (11- 12) and the vector of contact forces $f$ along the motion, and then solve the following optimization problem:

$$
\min _{\dot{S}, \mathbf{f}_{1}, \ldots, \mathbf{f}_{m}} f_{o b j}(\dot{S})
$$

s.t. at all $s=s_{i}$, for $1, \ldots, N$

$$
\begin{aligned}
\frac{d q}{d s} \dot{s} \in & {\left[\dot{q}_{\text {min }}, \dot{q}_{\text {max }}\right] } \\
\frac{d^{2} q}{d s^{2}} \dot{s}^{2}+ & \frac{d q}{d s} \ddot{s}+\left[\ddot{q}_{l o w}, \ddot{q}_{u p p}\right] \in\left[\ddot{q}_{\text {min }}, \ddot{q}_{\text {max }}\right] \\
a(s) \ddot{s}+ & b(s) \dot{s}^{2}+c(s)+M(q)\left[\ddot{q}_{\text {low }}, \ddot{q}_{u p p}\right] \\
& -\sum_{i} J_{i}(q)^{T} f_{i} \in\left[\tau_{\text {min }}, \tau_{\text {max }}\right] \\
m_{o b j} \alpha_{L}= & \sum_{i} f_{i}+m_{o b j} g \\
I_{o b j} \alpha_{A}= & \sum_{i}\left(p_{i}-c\right) \times f_{i} \\
A_{i} f_{i} \leq & 0 \text { for } i=1, \ldots, m
\end{aligned}
$$

For this each $\mathbf{f}_{i}$ is the stacked vector of contact forces at collocation point $i$, containing $3 m$ entries in total. As a result, this naïve formulation has $(1+3 m) N$ parameters and $3 n N$ constraints. This could be extremely expensive at high values of $m$ and $N$.

We present an alternate hierarchical method that decomposes this large problem into an inner problem embedded into an outer problem, both of which are simpler than the formulation above. It uses the fact that for each value of $s$ along the motion, contact forces may be chosen independently of one another. So, at each constraint evaluation point, a solution to (3), 6, (13) and (7) can be solved independently 
via a relatively small linear program to verify whether the given values of $\dot{s}$ and $\ddot{s}$ are valid:

Given $\dot{s}$ and $\ddot{s}$, find $f=\left(f_{1}, \ldots, f_{m}\right)$ such that

$$
\begin{aligned}
\sum_{i} J_{i}(q)^{T} f_{i} & \leq\left(a(s) \ddot{s}+b(s) \dot{s}^{2}+c(s)\right)+e_{\text {low }}-\tau_{\text {min }} \\
-\sum_{i} J_{i}(q)^{T} f_{i} & \leq \tau_{\max }-\left(a(s) \ddot{s}+b(s) \dot{s}^{2}+c(s)\right)-e_{u p p} \\
m_{o b j} \alpha_{L} & =\sum_{i} f_{i}+m_{o b j} g \\
I_{o b j} \alpha_{A} & =\sum_{i}\left(p_{i}-c\right) \times f_{i} \\
A_{i} f_{i} & \leq 0 \text { for } i=1, \ldots, m
\end{aligned}
$$

where $\left[e_{l o w}, e_{u p p}\right]$ is the interval resulting from the product $M(q)\left[\ddot{q}_{l o w}, \ddot{q}_{u p p}\right]$.

The outer optimization problem becomes:

$$
\begin{aligned}
& \text { Find } \dot{S}^{*}=\underset{\dot{S}}{\operatorname{argmin}} f_{o b j}(\dot{S}) \text { such that } \\
& \qquad \begin{aligned}
\text { for } i & =1, \ldots, N \\
\dot{q}_{\text {min }} & \leq 11 \leq \dot{q}_{\text {max }} \\
\ddot{q}_{\text {min }} & \leq 12 \leq \ddot{q}_{\text {max }} \\
& 15 \text { is satisfied }
\end{aligned}
\end{aligned}
$$

This approach reduces the number of outer optimization variables to $N$ and each iteration of the outer optimization requires solving $N$ independent inner LPs (15). Each LP requires solving for relatively few variables $(3 \mathrm{~m})$ and can be solved quickly. Overall, the hierarchical optimization is much faster than the naïve method, even for large number of contact points.

But this approach has a problem that, as formulated, the LP constraint is a black box binary test, which cannot be handled easily in numerical optimization routines that require the constraints to be differentiable with respect to $\dot{S}$. We use a formulation for turning this constraint into a piecewise linear, numerical form.

Let us rewrite [15] in a standard LP form:

$$
\begin{aligned}
& \text { Given } \dot{s} \text { and } \ddot{s} \text {, find } f=\left(f_{1}, \ldots, f_{m}\right) \text { such that } \\
& \qquad W f \leq z(\dot{s}, \ddot{s})
\end{aligned}
$$

where $W$ includes the linearized friction cone constraints $A_{i}$ and the Jacobian $J_{i}(q)^{T}$ which transforms the contact force into joint torques. $z(\dot{s}, \ddot{s})$ corresponds to the RHS of the inequalities in (15). By introducing an auxiliary variable $v$, we can transform this into a minimization problem whose solution is negative if and only if there is a solution to (15). We define the optimization variable $x=\left(f_{1}, \ldots, f_{m}, v\right)^{T}$ and formulate the objective function as $C x$ where $C=(0, \ldots, 0,1)$. The linear program becomes:

$$
\begin{array}{r}
\text { Solve } v^{*}(\dot{s}, \ddot{s})=\min _{x} C x \text { such that } \\
\\
W f-z(\dot{s}, \ddot{s}) \leq v \overline{1}
\end{array}
$$

where $\overline{1}$ is the vector of all $1 \mathrm{~s}$. The optimal value can be determined using a standard LP algorithm. We now replace the constraint at the outer level optimization with the continuous, piecewise-linear constraint $v^{*}(\dot{s}, \ddot{s}) \leq 0$. We can determine the subderivatives $\frac{\partial v^{*}}{\partial \dot{s}}$ and $\frac{\partial v^{*}}{\partial \ddot{s}}$ analytically through Karush Kuhn Tucker (KKT) multipliers of the LP (see Appendix). Using this formulation we are able to solve the outer optimization using standard nonlinear programming (NLP) techniques like sequential quadratic programming (SQP).

\section{Initial Guess}

Although it has been proven that the minimum-time timescaling problem as formulated is convex [15, 28], it is useful to obtain a good initial solution to speed up the outer optimization. To do so we maximize the velocities $\dot{S}$ of the time scaling function pointwise, so we compute the maximum possible $\dot{s}^{0}$ as our initial guess according to the constraint $\dot{q}_{\min } \leq \dot{q}=\frac{d q}{d s} \dot{s} \leq \dot{q}_{\max }$. Since the bounds $\left(\frac{d q}{d s}\right)_{\min }$ and $\left(\frac{d q}{d s}\right)_{\max }$ can be computed explicitly from the input path, we get $\left[\left(\frac{d q}{d s}\right)_{\min },\left(\frac{d q}{d s}\right)_{\max }\right] \dot{s} \in\left[\dot{q}_{\min }, \dot{q}_{\max }\right]$, then $\dot{s}^{0}$ can be approximated as:

$$
\dot{s}^{0}=\min \left(\min _{i}\left(\left|\frac{q_{\max }^{i}}{\left(\frac{d q^{i}}{d s}\right)_{\max }}\right|\right), \min _{i}\left(\left|\frac{q_{\min }^{i}}{\left(\frac{d q^{i}}{d s}\right)_{\min }}\right|\right)\right)
$$

where $i=1, \ldots, n$ iterates through each joint of the robot.

\section{E. Multiple Contact Phases}

In the presentation above, we assumed only one contact phase (no contacts are made or broken). But our method can handle situations with $N_{p}>1$ contact phases with a small modification. Each contact phase is distinguished by the set of contact points $F_{p}$ and friction coefficients, leading to different constraints of (13) and (6). We model the time-scaling as an optimization over $k$ piecewise quadratic function as in section IV-B, so the objective function in $[16$ becomes:

$$
f_{o b j}(\dot{S})=\sum_{p=1}^{N_{p}} \sum_{i=0}^{k} \frac{\Delta s_{p}}{\dot{s}_{p, i}+\dot{s}_{p, i+1}}
$$

where $\Delta s_{p}$ is the uniform interval in $p^{\text {th }}$ contact phase.

Since feasibility at a single collocation point only depends on the two optimization variables $\dot{s}_{i}$ and $\dot{s}_{i+1}$ of appropriate contact phase, no changes need to be made for the constraints formulated in [16].

\section{ITERATIVE LEARNING UNCERTAINTIES}

The second stage of our method is an iterative learning technique for improving confidence intervals given execution feedback. A conservative estimate of friction and execution errors could overly constrain the problem and generate a very slow motion, while an optimistic estimate could cause infeasibility. To approach the "sweet spot" we present two methods. The first method uses binary success/failure feedback to perform a bisection search on a chosen modeling parameter, (e.g., friction). Bisection continues until a desired convergence threshold is reached. This approach is most suitable 
for unobservable parameters like friction. The second method incorporates feedback on execution velocity and acceleration to measure confidence intervals more accurately.

\section{A. Bisection Search on Friction Coefficient}

Ideally, the optimized trajectory should move the blocks as fast as possible without tipping them over, but this is difficult to achieve due to modeling uncertainties. Instead, what we can achieve is to produce an trajectory that is $\epsilon$-near the timeoptimal trajectory, where $\epsilon$-near implies that the generated trajectory can be executed successfully, while executing the trajectory sped up by a factor $1+\epsilon$ fails. Here $\epsilon$ controls how close the generated motion approaches the true (unobservable) feasibility boundary of the fastest possible motion. A smaller $\epsilon$ indicates faster executions with lower margin of error, at the expense of more learning iterations.

Beginning from the initial confidence interval on the parameter of interest, a bisection search is conducted to find a lower bound that is nearly optimal according to the speed-up parameter $\epsilon$. We apply this to the coefficient of friction (COF). In manipulation it controls the perpendicular magnitudes of the contact forces and therefore affects the maximum accelerations applied on the object. It is also a difficult parameter to estimate accurately without specialized sensors.

The bisection search method (see Alg. 1) works by setting upper and lower bounds, $\mu_{u p p}$ and $\mu_{\text {low }}$, on the COF value $\mu$ and optimizing the problem formulated in 16 with $\mu$. With the assumption that the execution is relatively accurate or the error is negligible, we set the acceleration confidence interval to be empty, namely $\ddot{q}_{l o w}=\ddot{q}_{u p p}=0$. The optimized motion is executed, and if it fails (blocks tip over or shift largely beyond a predefined threshold), then we update $\mu_{u p p}$ with the current COF value $\mu$ and repeat. Otherwise, we update the lower bound on $\mu$. We then execute a sped-up version of this trajectory. If the sped-up execution fails, then we have converged successfully to a $\epsilon$-near optimal trajectory. We can also terminate if the difference between upper and lower bounds becomes less than a given tolerance $\delta(\delta=0$ is an acceptable value).

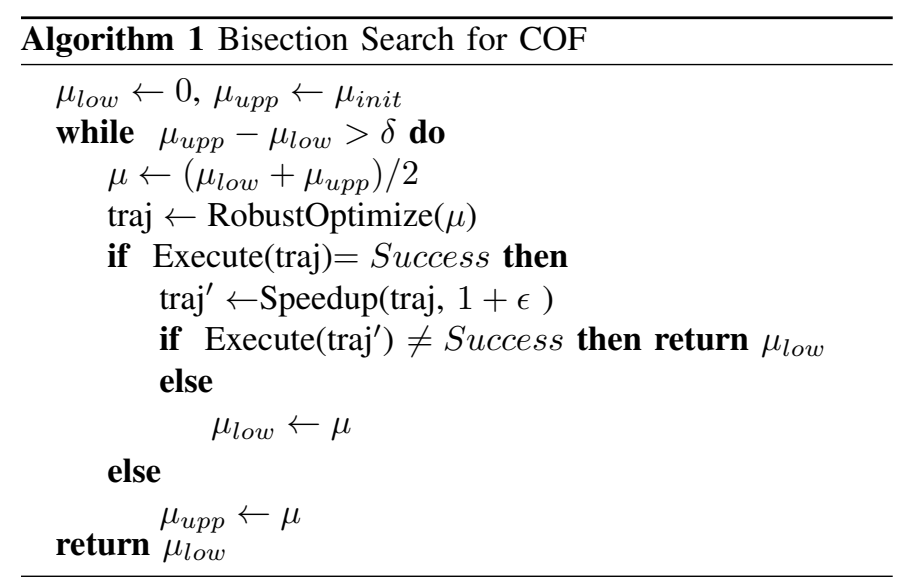

\section{B. Iterative Learning with Robust Optimization and Binary Search}

To consider errors on both parameter estimation and execution, we modify the binary search method for the modeling parameter in Sec. $\mathrm{V}$-A by adding an inner loop that learns and bounds execution disturbances via trajectory feedback.

To some extent, naïve introduction of measured confidence intervals into optimization can compensate for these disturbances. However, we have observed that many disturbances are trajectory-dependent (e.g., speed-dependent control errors, air resistance, etc.) Again we find that conservative estimates of errors $e$ with a large standard deviation $\Delta$ lead to slower optimized trajectories. Hence, our method learns characteristic disturbances during the iterative learning phase.

Our approach optimizes the trajectory with confidence interval $\left[\ddot{q}_{l o w}, \ddot{q}_{u p p}\right]$ from the last iteration, and update this confidence interval from the current execution feedback. This process repeats until the confidence interval becomes stable. Here stability is judged if the differences between each endpoint of the confidence interval for the last iteration and the current one become falls below a threshold. To estimate a per-trajectory confidence interval, we assume that for each time step the disturbance arises from a Gaussian noise model $N\left(U, \Delta^{2}\right)$.

Our algorithm Iterative-Learning-with-RobustOptimization-and-Binary-Search (ILROBS) is shown in Alg. 2. The input $K$ (used in Eq. (9)) is a user-specified parameter indicating the desired number of standard deviations of disturbances to which the model should be robust. In other words, the expected likelihood of success $\Phi(K)$ where $\Phi$ is the cumulative distribution of the standard normal distribution; and so $K$ should be chosen according to the 68 - 95 - 99.7 Rule [6]. After each execution the algorithm computes the noise model $N\left(U, \Delta^{2}\right)$ from $\ddot{q}_{e x c}-\ddot{q}_{\text {plan }}$. These are then used to determine the acceleration confidence interval according to 9 for the given $K$.

The challenge in this algorithm is to guess whether a given execution failed due to an overestimated friction coefficient or an unlucky disturbance (or contrariwise, succeeded due to random luck). In the former, the COF estimate should be lowered, and in the latter, further execution feedback may be needed to accurately estimate the probability of success. If we have observed that a given trajectory has succeeded all of $N_{e}(K)=\lceil 1 /(1-\Phi(K))\rceil$ times, then we believe that the success rate is at least $\Phi(K)$. So, if any one of them fails, we bisect.

\section{EXPERIMENTS}

We first test how the number of collocation points affects the computation time. And we also conduct two experiments corresponding to the two situations introduced in Sec. V SNOPT [10] was used for solving the outer optimization problem and the GNU GLPK library [11] was used for the inner linear program. The computation was carried on a laptop with $2.9 \mathrm{GHz}$ processor and the time for trajectory optimization for all the physical robot experiments are within 10 seconds. 

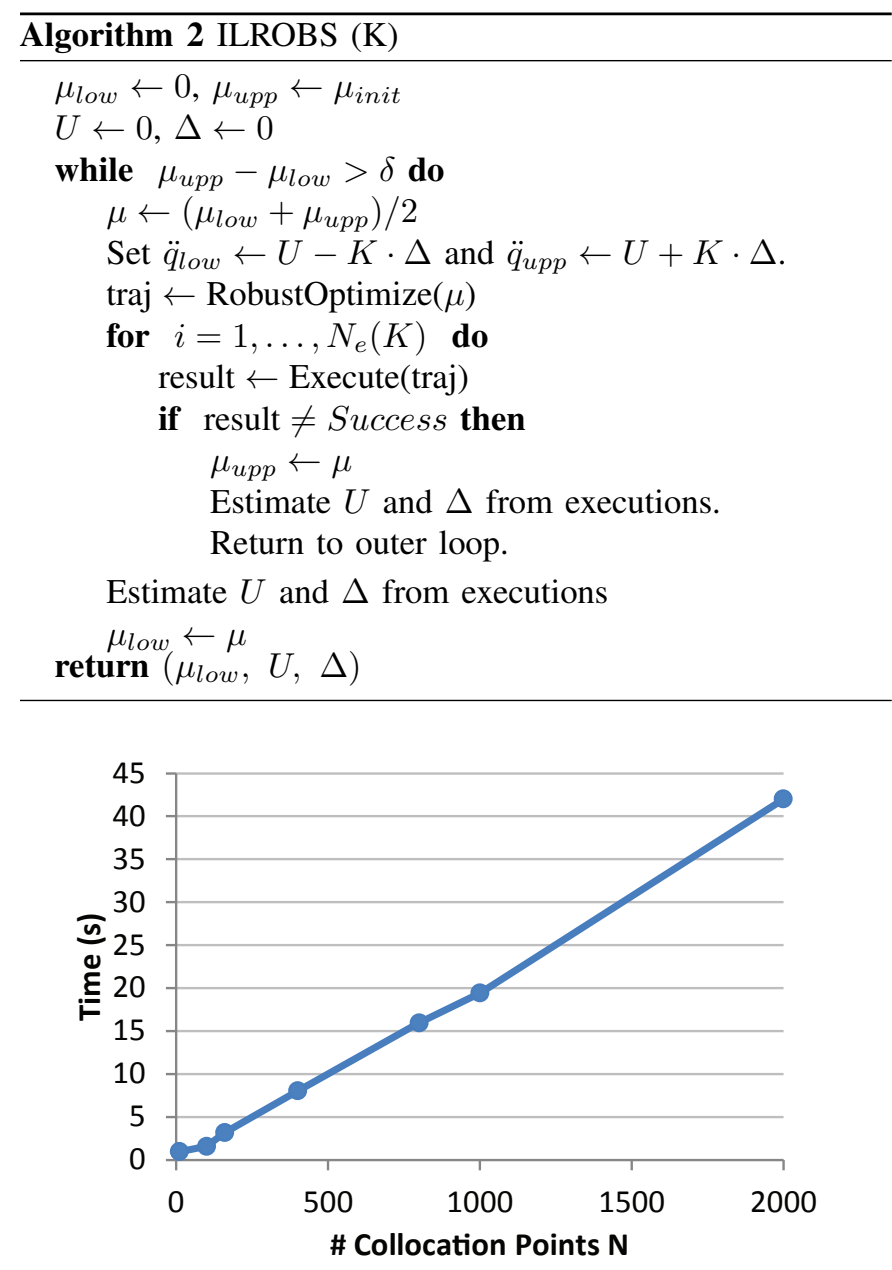

Fig. 3: Computation time versus number of time-domain collocation points for the two-block waiter task.

\section{A. Computation Time vs Number of Collocation Points}

The number of constraint checking points decides how many constraints must be evaluated and therefore affects the speed of optimization. We test the change of computation time in terms of the number of collocation points for the example of moving a stack of two blocks (Fig. 1. right). With 8 contacts points for the stack of two blocks, the computation time is shown in Fig. 3 for different number of collocation points. To keep computation times below $10 \mathrm{~s}$ per iteration, 200 collocation points are used throughout the following experiments.

\section{B. Binary Search COF with Hardware Execution}

To study the effect of friction uncertainty, the Stäubli TX90L industrial manipulator is used. A RobotiQ hand is installed on the manipulator to hold a plate with blocks on it. We wrap the contacting surfaces between blocks and plate with sand paper (Fig. 4). The COF is roughly estimated as $\mu=1$ by tilting the plate with the block on it and checking the inclination at which the block starts to slide.

First, the optimized trajectory with $\mu=1$ was too fast during execution on the physical robot, causing the objects to

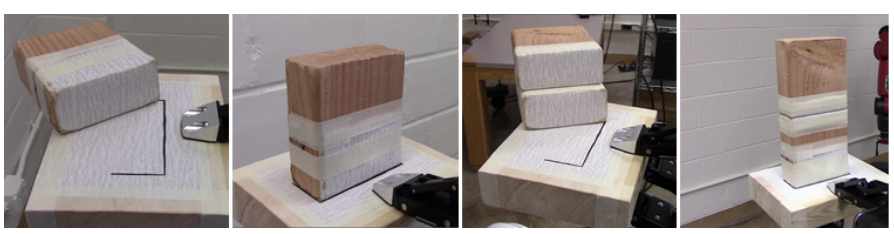

Fig. 4: The contact surfaces are wrapped with sand paper. One block and a stack of two blocks are placed on the plate shown on 2 nd and 4th picture respectively.

\begin{tabular}{|l|c|c|c|c|c|c|}
\hline Iter & $\mu$ & Exc. Time(s) & Rslt & Speedup Rslt & $\mu_{\text {upp }}$ & $\mu_{\text {low }}$ \\
\hline 1 & 1 & 3.229 & F & & 1 & 0 \\
\hline 2 & 0.5 & 3.232 & F & & 0.5 & 0 \\
\hline 3 & 0.25 & 3.704 & F & & 0.25 & 0 \\
\hline 4 & 0.125 & 5.237 & S & S & 0.25 & 0.125 \\
\hline 5 & 0.1875 & 4.276 & S & F & 0.25 & 0.1875 \\
\hline
\end{tabular}

TABLE I: Binary search on COF according to Alg. 1 for the example of moving a stack of two blocks. With $\epsilon=0.05$, the COF converges on an $\epsilon$-near optimal trajectory with $\mu=0.1875$ as in the highlighted row (i.e., speeding up the motion by $5 \%$ tips over the blocks).

slide in the one-block case and to wobble and fall over in the two-block case. Next, we applied the binary search method on COF to compensate for the un-modeled uncertainties which caused execution failure. Table $\mathbb{1}$ lists the binary search parameters for the two-block example. After five iterations it yields an $\epsilon$-near optimal value for COF and Fig. 5 shows the snapshots of the final motion execution.

We note that the converged parameter value $\mu=0.1875$ is less than a fifth of the empirically determined value of $\mu=1$. This is because the COF parameter acts as a proxy for all other un-modeled uncertainties, such as low-level controller errors and estimation errors in center-of-mass, contact points, etc. Curiously, the converged execution time of 4.276 is not far from the optimistic time of 3.229. This is because the optimization slows down the trajectory only where friction is the limiting constraint.

\section{Iterative Learning with Robust Optimization in Simulation}

In the second experiment, we introduce random disturbances during execution, and incorporate trajectory feedback into optimization as described in Sec. V-B. Because it is difficult to inject random disturbances to a real robot in the lab, we use a simulator for these examples. A rigid body simulator based on Open Dynamics Engine using a PID controller with feedforward gravity compensation torques [14] is used to track the planned trajectory $(t, q, \dot{q})$.

This example introduces both random disturbances and errors on model parameters. The optimization's initial COF estimate is 1 , while it is set to 0.5 in simulation. The uncertainties in trajectory execution are simulated by introducing random forces on the robot. A random horizontal force $F_{\text {disturb }}=(x, y, 0)$ with $x$ and $y$ subject to a Gaussian distribution $N(0,1)$ (in Newtons) is added to the tray at each simulation step (see Fig. 6).

The results of each outer loop of Alg. 2 are listed in Table [II. Here we run the algorithm with $K=1$ (corresponding to 


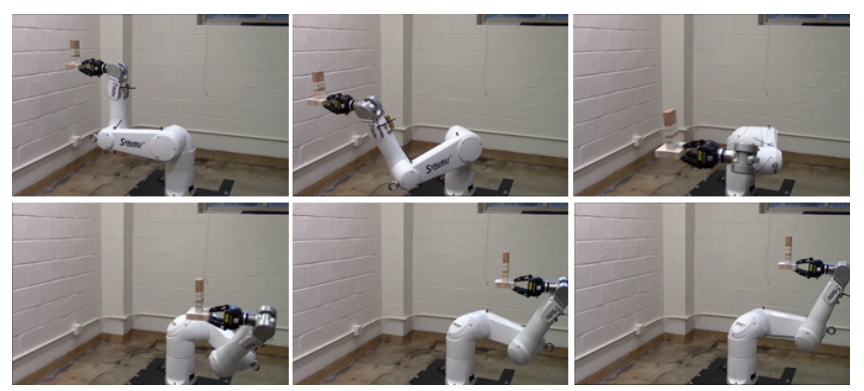

Fig. 5: Snapshots of executing the motion generated from Alg. 1 for moving a two-block stack.

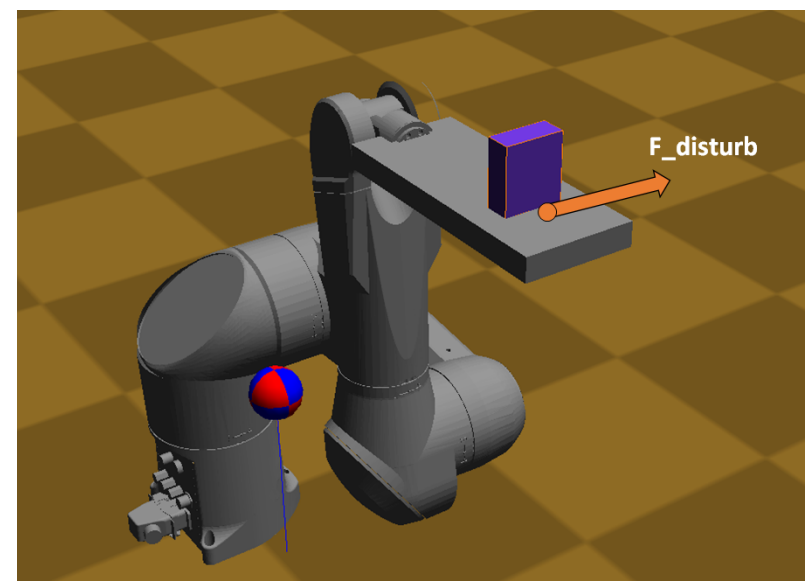

Fig. 6: In simulation tests, random horizontal forces with $2 \mathrm{~N}$ std. dev. are introduced on the tray at each simulation step.

a success rate of $68 \%$ ). Success ratios are estimated via 100 Monte Carlo trials. After 6 iterations, we learned the COF and a confidence interval of acceleration error that achieves a success rate of $74 \%$.

\section{CONCLUSION}

We present a fast time-scaling optimization method for generating time-optimal, dynamically feasible trajectory along a given geometric path for a robot in contact. By carefully formulating the problem as a non-linear optimization with the contact constraints as a linear program, we reduced the problem size and are able to solve the problem quickly (in seconds).

We apply this method on the time-optimal object moving task and we present two algorithms to handle the modeling and

\begin{tabular}{|c|c|c|c|c|c|}
\hline Iter & $\mu$ & Exc. Time(s) & Ratio(\%) & $\mu_{u p p}$ & $\mu_{\text {low }}$ \\
\hline 1 & 1 & 1.88 & 0 & 1 & 0 \\
\hline 2 & 0.5 & 2.20 & 0 & 0.5 & 0 \\
\hline 3 & 0.25 & 3.09 & 100 & 0.5 & 0.25 \\
\hline 4 & 0.375 & 2.52 & 98 & 0.5 & 0.375 \\
\hline 5 & 0.4375 & 2.30 & 74 & 0.5 & 0.4375 \\
\hline 6 & 0.46875 & 2.28 & 16 & 0.46875 & 0.4375 \\
\hline
\end{tabular}

TABLE II: Outer iterations of Alg. 2 for the one-block waiter task in simulation. With $K=1, \delta=0.05$, the algorithm converges in 6 iterations.
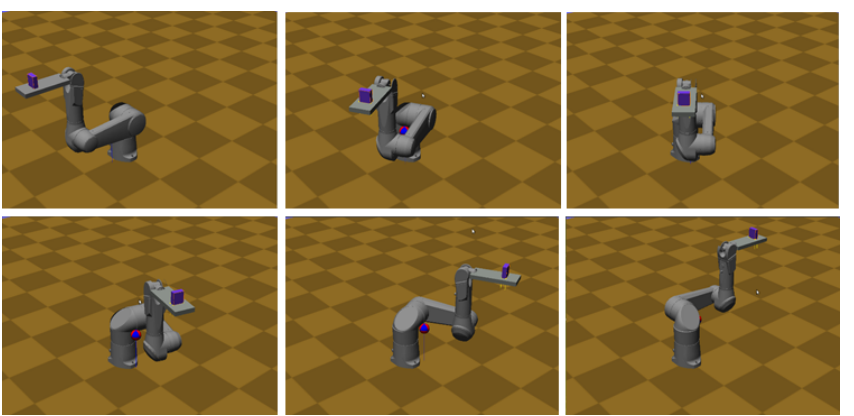

Fig. 7: Snapshots of successfully moving a block under disturbances in simulation.

execution uncertainties incorporating execution feedback. Our experiments demonstrate that this optimization method can be used with uncertainty learning process to generate motions that i) are near the optimum when there are modeling errors; and ii) can be executed multiple times with a success rate that depends on a user specified parameter.

There is substantial room for future work. First, we hope to extend our method to explicitly learn more than one independent hidden parameter, such as support shape, or inertial parameters. Another source of information may be the time of a detected failure; using this feedback may allow our method to learn more quickly. Finally, we hope to extend our method to optimize path shapes that are robust to error.

\section{APPENDIX}

Here we derive the formula for the derivative of the objective function of an inequality-constrained LP with respect to changes in the constraint RHS. Consider the parameterized LP:

$$
\min _{x} c^{T} x \text { such that } A x-b(t) \leq 0
$$

By the first-order KKT conditions, the optimal solution satisfies:

$$
\begin{aligned}
c+A^{T} \mu & =0 \\
A x^{*}-b & \leq 0 \\
\mu^{T}\left(A x^{*}-b\right) & =0 \\
\mu & \leq 0
\end{aligned}
$$

where $\mu$ is the vector of KKT multipliers (unrelated to the friction coefficient) and $x^{*}$ is the optimal solution. If the LP is bounded, $x^{*}$ lies at a corner point of the feasible region, and is defined by active constraints with $A_{i} x *-b_{i}=0$ and $\mu_{i}<0$. Denote $\hat{A}$ and $\hat{b}$ respectively as the matrix/vector containing the rows of $A$ and $b$ corresponding to active constraints, and let $\hat{\mu}$ be the set of active multipliers. Then:

$$
\begin{gathered}
c+\hat{A}^{T} \hat{\mu}=0 \\
\hat{A} x^{*}-\hat{b}=0
\end{gathered}
$$

with $\hat{A}$ an invertible matrix. So $x^{*}(t)=\hat{A}^{-1} \hat{b}(t)$, and $\frac{d}{d t}\left(c^{T} x^{*}\right)=c^{T} \hat{A}^{-1} \hat{b}^{\prime}(t)$. Since $\hat{\mu}^{T}=-c^{T} \hat{A}^{-1}$, we obtain $\frac{d}{d t}\left(c^{T} x^{*}\right)=-\mu^{T} \hat{b}^{\prime}(t)=-u^{T} \hat{b}^{\prime}(t)$ as desired. 


\section{REFERENCES}

[1] Brenna D Argall, Sonia Chernova, Manuela Veloso, and Brett Browning. A survey of robot learning from demonstration. Robotics and Autonomous Systems, 57 (5):469-483, 2009.

[2] Dimitris Bertsimas and Aurélie Thiele. Robust and data-driven optimization: Modern decision-making under uncertainty. INFORMS Tutorials in Operations Research: Models, Methods, and Applications for Innovative Decision Making, 2006.

[3] John T Betts. Survey of numerical methods for trajectory optimization. Journal of guidance, control, and dynamics, 21(2):193-207, 1998.

[4] J. E. Bobrow, S. Dubowsky, and J.S. Gibson. Timeoptimal control of robotic manipulators along specified paths. The International Journal of Robotics Research, 4(3):3-17, 1985.

[5] Douglas A Bristow, Marina Tharayil, and Andrew G Alleyne. A survey of iterative learning control. Control Systems, IEEE, 26(3):96-114, 2006.

[6] George W Cobb, Jeffrey A Witmer, and Jonathan D Cryer. An Electronic Companion to Statistics. Cogito Learning Media New York, 1997.

[7] D. Constantinescu and E. A. Croft. Smooth and timeoptimal trajectory planning for industrial manipulators along specified paths. J. of Robotic Systems, 17:223249, 2000.

[8] O. Dahl and L. Nielsen. Torque limited path following by on-line trajectory time scaling. In IEEE Int. Conf. on Robotics and Automation (ICRA), pages 1122-1128 vol.2, May 1989. doi: 10.1109/ROBOT.1989.100131.

[9] Adrien Escande, Abderrahmane Kheddar, Sylvain Miossec, and Sylvain Garsault. Planning support contact-points for acyclic motions and experiments on hrp-2. In Experimental Robotics, pages 293-302. Springer, 2009.

[10] Philip E. Gill, Walter Murray, Michael, and Michael A. Saunders. Snopt: An sqp algorithm for large-scale constrained optimization, 1997.

[11] GNU. Gnu linear programming kit (glpk). URL http: //www.gnu.org/software/glpk/glpk.html. (accessed April $16,2015)$.

[12] K. Harada, K. Hauser, T. Bretl, and J.-C. Latombe. Natural motion generation for humanoid robots. In IEEE/RSJ Int. Conf. on Intelligent Robots and Systems (IROS), 2006.

[13] Charles R Hargraves and Stephen W Paris. Direct trajectory optimization using nonlinear programming and collocation. Journal of Guidance, Control, and Dynamics, 10(4):338-342, 1987.

[14] Kris Hauser. Robust contact generation for robot simulation with unstructured meshes. In International Symposium on Robotics Research, Singapore, 2013.

[15] Kris Hauser. Fast interpolation and time-optimization with contact. The International Journal of Robotics
Research, 33(9):1231-1250, 2014.

[16] Puttichai Lertkultanon and Quang-Cuong Pham. Dynamic non-prehensile object transportation. In Int. Conf. on Control Automation Robotics Vision (ICARCV), pages 1392-1397, Dec 2014.

[17] C Karen Liu. Dextrous manipulation from a grasping pose. ACM Transactions on Graphics (TOG), 28(3):59, 2009.

[18] Jingru Luo and K. Hauser. Interactive generation of dynamically feasible robot trajectories from sketches using temporal mimicking. In IEEE Int. Conf. on Robotics and Automation (ICRA), pages 3665-3670, may 2012.

[19] Kevin M Lynch and Matthew T Mason. Dynamic underactuated nonprehensile manipulation. In IEEE/RSJ Int. Conf. on Intelligent Robots and Systems (IROS), volume 2, pages 889-896. IEEE, 1996.

[20] Igor Mordatch, Zoran Popović, and Emanuel Todorov. Contact-invariant optimization for hand manipulation. In Proceedings of the ACM SIGGRAPH/Eurographics symposium on computer animation, pages 137-144. Eurographics Association, 2012.

[21] Duy Nguyen-Tuong and Jan Peters. Model learning for robot control: a survey. Cognitive processing, 12(4):319_ 340, 2011.

[22] Quang-Cuong Pham, Stéphane Caron, Puttichai Lertkultanon, and Yoshihiko Nakamura. Planning truly dynamic motions: Path-velocity decomposition revisited. arXiv preprint arXiv:1411.4045, 2014.

[23] Michael Posa and Russ Tedrake. Direct trajectory optimization of rigid body dynamical systems through contact. In Workshop on the Algorithmic Foundations of Robotics, 2012.

[24] Michael Posa, Cecilia Cantu, and Russ Tedrake. A direct method for trajectory optimization of rigid bodies through contact. The International Journal of Robotics Research, 33(1):69-81, 2014.

[25] Stefan Schaal and Christopher G Atkeson. Learning control in robotics. Robotics \& Automation Magazine, IEEE, 17(2):20-29, 2010.

[26] Kang Shin and N. McKay. Minimum-time control of robotic manipulators with geometric path constraints. IEEE Trans. on Automatic Control, 30:531-541, 1985. doi: 10.1109/TAC.1985.1104009.

[27] J.-J.E. Slotine and H.S. Yang. Improving the efficiency of time-optimal path-following algorithms. IEEE Trans. on Robotics and Automation, 5(1):118 -124, Feb 1989. ISSN 1042-296X. doi: 10.1109/70.88024.

[28] D. Verscheure, B. Demeulenaere, J. Swevers, J. De Schutter, and M. Diehl. Time-optimal path tracking for robots: A convex optimization approach. IEEE Trans. Automatic Control, 54(10):2318 -2327, October 2009. ISSN 0018-9286. doi: 10.1109/TAC.2009.2028959.

[29] Oskar von Stryk and Roland Bulirsch. Direct and indirect methods for trajectory optimization. Annals of Operations Research, 37(1):357-373, 1992. 